\title{
THE PSYCHOLOGICAL BACKGROUND OF MASTICATORY SYSTEM PARAFUNCTIONS
}

\author{
Tamara Pawlaczyk-Kamieńska \\ Department of Pediatric Dentistry, Poznan University of Medical Sciences, Poland
}

\begin{abstract}
In recent years there has been a growth noted in the number of people suffering the effects of the masticatory organ motoric disorders which arise as a consequence of parafunctions. Some of them accompany mental disorders where practising parafunctions may ease anxiety and stress. Unfortunately, these parafunctions may produce injuries in the mouth. Moreover, they strain the motor system of the masticatory organ unevenly. This may lead to formation and fixation of a deviated model of function and, with time, to morphological and functional changes. Identification and elimination of a causative factor is essential in undertaking proper and effective treatment of these disorders. Moreover, it is necessary to promote parafunctions prophylaxis and to provide comprehensive, team-based treatment. It seems purposeful to implement comprehensive therapy at an early stage of the problem as a part of psychologic treatment, too. It should aim at eliminating harmful habits resulting in secondary somatic complications. The paper presents current views on etiology of masticatory organ parafunctions.
\end{abstract}

KEY WORDS: masticatory organ, motoric disorders, parafunctions, mental disorders.

J Stoma 2018; 71, 6: 507-510

DOI: https://doi.org/10.5114/jos.2018.85569

Parafunctions are motor stereotypes having the character of habits which are not related to any physiological processes or mechanisms. These are, therefore, all types of acquired habits, most usually practised by a patient involuntarily. Effects of such behaviour are visible in the mouth and may contribute to soft tissue and teeth injuries [1-4]. According to the International Statistical Classification of Diseases and Related Health Problems ICD-10 parafunctions may be classified as predominantly compulsive acts (F42.1) or other habit and impulse disorders (F63.8) [5]. The term "parafunctions" in dentistry is usually applied to describe habits affecting the masticatory system, which is a morphological-functional system associated with the digestive tract. The masticatory system is composed of the teeth, their supporting structures, the bones of the maxilla and the mandible, the temporomandibular joints (TMJ), the muscles involved directly or indirectly in mastication, and the vascular and nervous systems supplying these tissues. Functional and structural disturbances in any of the components of this system may be reflected by functional or structural disorders in one or more of its other components. The lesions may also affect as remote muscles as those found in e.g. the neck, the back of the neck, shoulder girdle, and even the upper limb or the chest $[1,4,6,7]$.

Oral parafunctions of the masticatory organ may be classified into two groups:

1. Acquired - those are the habits considered as physiological reflexes in infants. A child learns them at first but while gaining body maturity, especially nervous system maturity, should give them up. However, if

JOURNAL OF STOMATOLOGY CZASOPISMO STOMATOLOGICZNE

AdDress FOR CORRESPONDEnce: Tamara Pawlaczyk-Kamieńska, DDS, PhD, Department of Pediatric Dentistry, Poznan University of Medical Sciences, 70 Bukowska St., 60-812 Poznań, Poland, phone: +48 6185470 53, fax: +48 6185470 59, e-mail: tamara_pawlaczyk@wp.pl 
the reflex persists it is turned into a habit. Sometimes a given habit is replaced with another one.

2. Compulsive - these are established habits which appear when an emotional tension is stronger than a child's adaptive abilities. By practising the habit a child feels safer [8].

In dentistry parafunctions are classified considering contacts with the teeth during activities not related to chewing. There are:

1. Occlusal parafunctions - occurring when surfaces of the opposing teeth meet together:

- bruxism;

2. Non-occlusal parafunctions - occurring when the opposing teeth do not come in contact; a patient has a habit of inserting fingers or other foreign bodies between the teeth:

- finger sucking,

- onychophagia.

Non-occlusal parafunctions also include: biting on the labial, lingual or buccal mucosa, lip sucking, tongue thrusting, sucking or biting on various objects (needles, pencils, pens).

Due to parafunctions the masticatory organ is unevenly strained, which may produce and establish an improper activity model and, with time, lead to formation of morphological and functional changes [3, 6, 9-11].

Digitomania is a retained habit of finger sucking (from Greek digitus - a finger). Sucking reflex is a physiological adaptive reflex typical of mammals. Apart from the rooting reflex, it is one of the most developed reflexes of an infant, allowing it to survive. With the growing maturity of the central nervous system the sucking reflex disappears. After two years of age a child should suck on a finger significantly less often. At this age it will suck on a finger when sleepy, tired, or while falling asleep. If the sucking habit persists over the third year of age it is considered a non-physiological, habitual activity $[3,11]$.

Finger sucking in children may impair proper development of the masticatory organ and cause changes in morphology, thus producing malocclusion. The impact of finger sucking on a child's masticatory organ depends on its age, intensity and duration of the habit, and the way a finger is placed in the mouth. The most frequently observed effect is a restriction in vertical growth of the maxillary alveolar process and the alveolar part of the mandible, resulting in an open bite. Other complications may include mandibular prognathism, protrusion of the upper incisors and retrusion of the lower ones, as well as an increase in the horizontal overjet above 3-4 $\mathrm{mm}[3,11]$.

Finger sucking may also be a cause of oral mucosa injuries. Moreover, fingers may be the carriers of pathogens of the Enterobacteriaceae (e.g. Escherichia coli, Salmonella spp., Shigella spp., Yersinia spp., Klebsiella spp., Enterobacter spp.) $[9,11,12]$. The sucking habit, through thrusting the tongue between the dental arches, may predispose to the development of faulty pronunciation, impaired chewing or a retained neonatal type of swallowing [11]. Still, the signs of exercising this parafunction do not only affect the masticatory organ. In thumb sucking children thumb deformations, skin lesions, ulcerations and paronychia are observed. Moreover, a persisting habit of finger sucking may lead to formation of other harmful habits such as lip or cheek sucking, tongue biting or pressing, and pencil or other foreign objects sucking $[11,13]$.

Finger sucking may be substituted by nail biting, called onychophagia. The term derives from Greek onycho - "a nail" and phagos - "to eat", together meaning the biting and eating the nails. As nail biting mostly concerns hand nails it is relatively easy to diagnose by observing the patient's hands. In some people this activity may develop into other habits such as lip or pencil biting, or winding the hair on fingers [3, 11-15].

Onychophagia is classified into the obsessive-compulsive spectrum disorders and may be due to hyperirritability (stress, excitation, anxiety) or, just the opposite, to hypostimulation (boredom, lack of activity) [12, 14, 15]. This parafunction occurs mainly in childhood, but in some cases it may continue into adulthood. Most epidemiological studies are limited to children or adolescents. The prevalence of onychophagia during childhood and adolescence ranged from $20 \%$ to $33 \%$ [12]. Children often bite their nails when they learn for school, especially if they are having a problem with their homework, and while they read or watch sad or scary films [13]. If this parafunction affects people in the growing age it may not only contribute to tooth wearing and overloading but also to an uneven load directed on the temporomandibular joint and to occlusion defects, thus causing impaired development of the masticatory organ. The effects of practising parafunctions are related to the habit duration and intensity, and the age of a child (the stage of the masticatory system development). Depending on the site in the mouth of nail biting, various occlusal defects are observed: open anterior bite, right or left lateral bite, mandibular dislocation to one side, e.g. to the right, resulting from biting the nails of both the right and left hand on the right side, only $[3,7,13]$.

The majority of nails biters do it absolutely unwittingly, usually while performing other activities. However, there are patients who quit other activities consciously to bite their nails. They do it purposefully and willingly. Many people affected by onychophagia describe it as a barely resistible compulsion. Some add that they feel pleasure and relaxation $[12,14]$. There are people, too, who bite the nails (or hangnails) to even out all imperfections and provide an ideal shape to them. Their onychophagia is practised out of the necessity to be perfect [3, 12-15].

The most frequent effect of this parafunction is hand aesthetics, although it may also be the cause of skin and dental disorders. Dermatological abnormalities due to onychophagia include nail platelesions of various severity, paronychia, secondary bacterial infection of the dam- 
aged areas around the nail, detachment of the nail from its bed, and irreversible shortening of the nails $[12,15]$.

The habit of nail biting, similarly to finger sucking, may be the route through which numerous microorganisms are introduced to the mouth $[9,11]$. Oral lesions may affect soft tissues (injuries, damage to the gums) and teeth (incisal edge wear of the anterior teeth). Damage to teeth due to nail biting belongs to non-carious lesions, i.e. arising without the contribution of cariogenic microorganisms. It is referred to as teeth abrasion, pathological wear of the tissues resulting from contact of teeth with exogenic materials. Such wear is observed in a single tooth or a group of teeth which, if left untreated, may produce complications and eventually a tooth loss. This not only leads to bad looks, but most importantly to the instability of the masticatory system $[13,16,17]$.

Nail biting may also affect daily life and have psychological consequences. Nail biters sometimes hide their hands out of shame, which may lead to limiting their social contacts $[12,14]$.

Bruxism is considered the most destructive parafunction. It is defined as a repetitive jaw-muscle activity characterized by clenching or grinding of the teeth and/ or by bracing or thrusting of the mandible. It may co-exist with other parafunctions e.g. with nail biting, especially in younger people [18-21].

There are two types of bruxism, which may occur separately and together:

- awake bruxism (AB),

- sleep bruxism (SB).

Awake bruxism occurs during wakefulness and is usually seen as a jaw clenching habit that appears in response to stress and anxiety. Patients may notice their habit, be aware of it and control it to some extent. Frequently, teeth clenching accompanies focusing attention on a performed activity. The habit may also accompany an intensive emotional tension. Stressful situations contribute to the growth in the sensitivity of receptors in the muscles. With an excessive stimulation, they produce an uncontrolled and increased contraction of the masseter muscles. In adults he prevalence of awake bruxism is about 30\% [3, 18-22].

Sleep bruxism presents as an involuntary, audible by other people, teeth grinding and clenching, both in habitual maximum intercuspation and eccentric position. It is a parafunction practised without a patient's conscience. The prevalence of sleep bruxism is about $15 \%$ [22]. Currently, sleep bruxism is considered not to be related to psychogenic factors. It is classified into the category of parasomnias, i.e. sleep disorders involving abnormal movements or behaviours of unknown origin $[19,20,23,24]$. It is believed that parasomnias manifest central nervous system activation and appear between sleep stages or during arousal from sleep. Parasomnias also include somnambulism, sleep hallucinations, nightmares, somniloquy (sleep talking), and restless legs syndrome. Bruxism is listed in the International
Classification of Sleep Disorders (ICSD) and is third most common form of sleep disorder after sleep talking and snoring $[18,20,23-25]$.

Bruxism is diagnosed on the basis of a person's history, clinical signs and symptoms and instrumental techniques [20]. Lobbezoo et al. [20] suggested that on the basis of self-report (questionnaires or the anamnestic part of clinical examination) "possible" sleep or awake bruxism can be diagnosed. Based on self-report and the inspection part of a clinical examination, "probable" SB or $\mathrm{AB}$ can be diagnosed. And based on self-report, a clinical examination and instrumental techniques, such as electromyography (EMG), "definite" awake bruxism, or polysomnography (PSG) "definite" sleep bruxism can be diagnosed [20].

Dental clinical examination shows pathological tooth wear leading to crown height loss and morphological changes of its incisal edges and/or chewing surfaces. It is tooth attrition, wear of hard dental tissues caused by excessive contacts of the opposing teeth (tooth-tooth), without the involvement of foreign objects. The lesions are found on those dental surfaces which participate in occlusion or articulation. However, clenching during awake bruxism does not produce their wear $[1,3,10$, $19,22,24]$. Another clinically diagnosed sign of bruxism is abfraction, the loss of cervical tooth structure in the absence of caries. Due to excessive forces affecting the chewing surfaces during their contact with opposing teeth microfissures may form, especially in the enamel, which is less flexible than dentin, with lower tensile strength $[10,24]$. Apart from objective signs visible in the clinical examination, the patient may complain of symptoms such as pain in the masticatory and cervical muscles, pain in the TMJ, tension headache, painful neck, back, throat, shoulders, and tension headaches, hypersensitive teeth or excessive tooth mobility. Moreover, patients may report numbness, paraesthesia of the facial skin, ears, and shoulders, stiff neck, pains in the spinal column, tinnitus, vision abnormalities, sleep abnormalities, general emaciation, chronic fatigue, and mental discomfort [3, 7, 19-21, 24-26].

The etiology of bruxism is multifactorial and includes peripheral factors such as tooth interference in dental occlusion, psychosocial influences such as stress or anxiety and central or pathophysiological causes involving brain neurotransmitters or basal ganglia [22-24, $27,28]$. AB is currently considered to be a parafunctional reaction to mental or physical stress and SB may be a pathological variant of normal physiological activity $[27,28]$.

The etiology of oral parafunctions is very complex. Both local and general factors are taken into consideration. However, currently this is a psychogenic factor related to increased emotional tension that is considered to play a major role $[17,22,26,27]$. Stressful situations predispose to practising parafunctions and affect their intensity; they are one of the ways to cope with stress. For 
some patients parafunctions seem to be compulsory; they cannot quit them [12, 22, 27]. Unfortunately, the result is increased muscle tension, especially of the masseter muscles, and overloading individual elements of the masticatory organ (TMJ, teeth, periodontium). The muscles may be sore, tender, and with time overgrown, resulting in a square face look $[3,6]$. What is more, as a result of dental hard tissue loss increased teeth sensitivity to chemical and thermal stimuli occurs. Then, a patient complains of teeth oversensitivity. Due to teeth overload and/or their wear, complications may occur, such as pulp diseases, teeth fractures, and the above-mentioned occlusion defects $[3,10]$. Parafunctions, mainly among people with decreased sufficiency of periodontium, may cause periodontopathies. On the other hand, in patients with properly functioning periodontium adaptive changes of the periodontium and alveolar process occur, visible as dense bone trabeculation in radiological examination. Parafunctions may also bring about injuries to the oral soft tissues $[3,12,13]$.

\section{CONCLUSIONS}

Parafunctions are diagnosed based on history, signs and symptoms. Their detection and awareness of their occurrence on the part of a patient are essential to eliminate a harmful habit. Unfortunately, these motor pathologies are a source of excessive forces exerted on dental surfaces, periodontium, and joints of the masticatory system, which produces the system strain, and changes in its morphology and function. Then, promotion of parafunctions prophylaxis as well as the introduction of comprehensive, team-based management including prophylaxis and treatment is necessary. Therefore, it seems appropriate in psychology and psychiatry, too, to implement comprehensive therapy in an early stage aiming at elimination of harmful habits causing secondary somatic complications.

\section{CONFLICT OF INTEREST}

The authors declare no potential conflicts of interest with respect to the research, authorship, and/or publication of this article.

\section{References}

1. Farsi NM. Symptoms and signs of temporomandibular disorders and oral parafunctions among Saudi children. J Oral Rehabil 2003; 30: 1200-1208.

2. Glaros AG, Hanson AH, Ryen CC. Headache and oral parafunctional behaviors. Appl Psychophysiol Biofeedback 2014; 1 : 59-66.

3. Kamdar RJ, Al-Shahrani I. Damaging oral habits. J Int Oral Health 2015; 7: 85-87.

4. Sena MF, Mesquita KS, Santos FR, et al. Prevalence of temporomandibular dysfunction in children and adolescents. Rev Paul Pediatr 2013; 31: 538-545.
5. World Health Organization. ICD-10 Classification of Mental and Behavioural Disorders: Diagnostic Criteria for Research. Geneva, 1993.

6. Franco-Micheloni AL, Fernandes G, de Godoi Gonçalves DA, Camparis CM. Temporomandibular disorders in a young adolescent Brazilian population: epidemiologic characterization and associated factors. J Oral Facial Pain Headache 2015; 29: 242-249.

7. Okeson JP. Management of Temporomandibular Disorders and Oclusion. $7^{\text {th }}$ ed. Mosby Elsevier, Missouri 2013.

8. Finn SB. Clinical Pedodontics. Saunders, Philadelphia 1998.

9. Baydas U, Uslu H, Yavuz I, et al. Effects of chronic nail biting habit on the oral carriage of Enterobacteriae. Oral Microbiol Immunol 2007; 22: 1-4.

10. Harpenau LA, Noble WH, Kao RT. Diagnosis and management of dental wear. J Calif Dent Assoc 2011; 39: 225-231.

11. Kamal FG, Bernard RA. Influence of nail biting and finger sucking habits on the oral carriage of Enterobacteriaceae. Contemp Clin Dent 2015; 6: 211-214.

12. Pacan P, Grzesiak M, Reich A, et al. Onychophagia and onychotillomania: prevalence, clinical picture and comorbidities. Acta Derm Venereol 2014; 94: 67-71.

13. Tanaka OM, Vitral RW, Tanaka GY, et al. Nailbiting, or onychophagia: a special habit. Am J Orthod Dentofacial Orthop 2008; 34: 305-308

14. Pacan P, Grzesiak M, Reich A, Szepietowski JC. Onychophagia as a spectrum of obsessive-compulsive disorder. Acta Derm Venereol 2009; 89: 278-280.

15. Krejci CB. Self-inflicted gingival injury due to habitual fingernail biting. J Periodontol 2000; 71: 1029-1031.

16. Williams TI, Rose R, Chisholm S. What is the function of nail biting: an analog assessment study. Behav Res Ther 2006; 45: 989-995.

17. Baad RK, Jagtap K. The study of role of stress in children with behavior disorders and orofacial lesions. J Contemp Dent Pract 2012; 13: 559-561.

18. Manfredini D, Lobbezoo F. Role of psychosocial factors in the etiology of bruxism. J Orofac Pain 2009; 23: 153-166.

19. Nahás-Scocate AC, Coelho FV, de Almeida VC. Bruxism in children and transverse plane of occlusion: is there a relationship or not? Dental Press J Orthod 2014; 19: 67-73.

20. Lobbezoo F, Ahlberg J, Glaros AG, et al. Bruxism defined and graded: an international consensus. J Oral Rehab 2013; 40: 2-4.

21. Ziółkowska-Kochan M, Kochan J, Pracka D, et al. Bruxism - a multidisciplinary problem. Czas Stomatol 2007; 40: 391-397.

22. Manfredini D, Landi N, Romagnoli M, Bosco M. Psychic and occlusal factors in bruxers. Aust Dent J 2004; 49: 84-89.

23. Lavigne G, Kato T, Kolta A, Sessle BJ. Neurological mechanisms involved in sleep bruxism. Crit Rev Oral Biol Med 2003; 14: 30-46.

24. Tsiggos N, Tortopidis D, Hatzikyriakos A, Menexes G. Association between self-reported bruxism activity and occurrence of dental attrition, abfraction, and occlusal pits on natural teeth. J Prosthet Dent 2008; 100: 41-46.

25. Ohayon MM, Li KK, Guilleminault C. Risk factors for sleep bruxism in general population. Chest 2001; 119: 53-61.

26. Townsen D, Nicholson RA, Buenaver L, et al. Use of a habit reversal treatment for temporomandibular pain in a minimal therapist contact format. J Behav Ther Exp Psychiatry 2001; 32: 221-239.

27. Calixtre LB, da Silva Grüninger BL, Chaves TC, de Oliveira AB. Is there an association between anxiety/depression and temporomandibular disorders in college students? J Appl Oral Sci 2014; 22: 15-21.

28. Yap AU, Chua AP. Sleep bruxism: Current knowledge and contemporary management. J Conserv Dent 2016; 19: 383-389. 